\title{
TOPIC: INGUINAL HERNIA - Post op chronic pain: incidence, evaluation, legal consequences, therapy, follow up
}

(C) Springer-Verlag 2014

\author{
VS:40 \\ FEMORAL NERVE INJURY AFTER TEP REPAIR: \\ EVALUATION OF 4 PATIENTS AND SURGICAL \\ APPROACH \\ M Ertem ${ }^{2}$, H Gök $^{1}$, E Hatipoglu² ${ }^{2}$ E Özveri ${ }^{1}$ \\ ${ }^{\prime}$ Acibadem Kozyatagi Hospital, Department of Surgery, \\ Istanbul, TURKEY \\ ${ }^{2}$ Istanbul Uni., Cerrahpasa School of Medicine, \\ Department of Surgery, Istanbul, TURKEY
}

Introduction: Nerve injury has a reported incidence of $2 \%$ during laparoscopic hernia repair. Femoral nerve injuries are seen less frequently than the femoral branch of the genitofemoral nerve and the lateral cutaneous nerve of the thigh. We are presenting videos of the this complication and want to discuss the reasons and solutions. In addition, we review the anatomy and technique in an effort to help avoid this complication in the future.

Methods: Here we present 4 patients whose fermoral nerves has been injured after TEP repair. One patient bilateral, one had right and the other two had left side hernia repaired, respectively. Typical symptom of the patients was pain and they had a specific walking which we called "duck style walking". Femoral hernia injury was documented by EMG at the all patients.

Results: We approached via transabdominal route. We removed most of the tacks and the meshes at the all patients. All the patients were discharged the day after operation. Pain scores of the patients were improved significantly.

Conclusion: There has been paramount concern among most surgeons over complications caused by staples in the form of nerve entrapment leading to chronic pain in laparoscopic inguinal hernia repair. Surgeons have been using $5 \mathrm{~mm}$ tacking devices for laparoscopic hernia repair. These devices penetrate tissue more deeply and in so doing may injure nerves not classically at risk, such as the ilioinguinal and the iliohypogastric. In our experience, the main reason of femoral hernia injury after TEP repair is making fixation on the triangle of pain. To avoid this complication, one must not make any fixation on the inferolateral part of the deep ring.

\author{
VS:41 \\ CORRECTION OF LAPAROSCOPIC BILATERAL \\ INGUINAL HERNIA WITH TEP IN AMBULATORY \\ WITH SELF-ADHERING PROSTHETICS \\ G Cardoso ${ }^{1}$, C Magalhães \\ 'Hospital Santo António (CICA) - CHP, Porto, PORTUGAL
}

Introduction: For the last three decades, the number of surgical procedures in an ambulatory regime has grown progressively for several reasons, which include the development of less invasive techniques, improvement in anesthetic techniques and control over post-surgery pain, increasing experience in medical and nursery teams during ambulatory, as well as the need to reduce the waiting list and lower the costs for the Health System

Methods: The authors presented the video of a clinical case about the correction of an inguinal hernia through laparoscopic approach with TEP using self-adhering prosthetics.

Results: The patient of male gender, admitted in ambulatory surgery appointment with a bilateral inguinal hernia and without comorbidities or surgical history, was subject to a correction of a bilateral inguinal hernia through laparoscopic with TEP. The procedure and post-surgery took place without complications, having the patient been discharged by $6 \mathrm{pm}$ the same say of the surgery procedure. The patient is currently without symptoms and without evidence of relapse.

Discution: The laparoscopic inguinal hernioplasty with TEP is a procedure that, although demands a wider learning curb, if executed by experienced surgeons, does not require a longer procedure time comparing to open surgery, presenting clear advantages for the patient in a sense that the patient has a less painful post-surgery, ultimately being able to return to his daily and working routine quicker. In the case of the TEP this presents the advantage of not violating the peritoneum, reducing the risks of injuring the intra-abdominal organs. This is a procedure that can be done in ambulatory surgery with discharge on the same say, once it presents a low complications rate, low conversion rate and high patient satisfaction rate.

The use of self-adhering prosthetics in laparoscopic/video assisted approaches can be a solution to the prosthetics fixation problem in the treatment for inguinal hernias. 